\title{
Gestische Praxis und sprachliche Form
}

\begin{abstract}
Dieser Beitrag ist ein Argument für die Subsumption grammatischer Analyse sprachlicher Formen unter die Analyse kommunikativer Praktiken. Er beschreibt zunächst ein Phänomen, das regelmäßig beschreibende Handgesten begleitet (der Sprecher blickt auf die eigene, gestikulierende Hand) und diskutiert dann sprachliche Einheiten (Wörter und Konstruktionen) in vier Sprachen (Deutsch, Japanisch, Ilokano und US-Englisch), die ebenfalls regelmäßig mit beschreibenden Handgesten verbunden sind und diese gleichsam in die Struktur der sprachlichen Äußerung integrieren bzw. das Bindeglied einer bimodalen Beschreibung bilden. Man kann diese bimodalen Gebilde als sprachspezifische Konstruktionen fassen, aber ebenso als Sedimente zunächst sprachunabhängiger Praktiken, die sich spezifischer einzelsprachiger Ressourcen bedienen. Demgegenüber lassen sich gestische Beschreibungen selbst in der Regel nur als improvisierende Realisierungen von Praktiken (gestischen Beschreibungsmethoden) auffassen, nicht aber als Formen in einem je schon existierenden Formsystem. Wie neue sprachliche Formen durch die Rekonfiguration kommunikativer Praktiken sedimentiert werden und wie Form und Praktik einander bedingen, wird am Beispiel des neuen US-Englischen verbum dicendi ,be like“ illustriert.
\end{abstract}

\section{Einleitung}

In ihrer Einführung in das Thema dieses Bandes weisen Deppermann/Feilke/ Linke (in diesem Band) wiederholt auf den eigentümlichen Zwischenstatus des Begriffs der Praktiken hin. Praktiken sind zum Beispiel irgendwo zwischen tradierten Formen und situativen Handlungen angesiedelt: Sie erklären die Rekurrenz der Formen und sind doch mit Formen nicht identisch. Sie schreiben, dass sich mit den Begriffen ,Praxis‘ und ,Praktik‘ das

Versprechen [verbindet], Sprache, Text, Verhalten und Kommunikation neu zu denken. Prozessualität, Verkörperung und soziale Routinen rücken ins Zentrum des Gegenstandsverständnisses. (ebd.)

In diesem Beitrag stelle ich dar, wie sich Praktiken bei meinen Versuchen, Handgesten gleichsam aus konversationsgrammatischer Perspektive zu analysieren, d.h. ihre Einbettung und Funktionen in sprachlichen Äußerungen zu erfassen, 
geradezu aufgedrängt haben, in dem Maße, in dem traditionellere Begriffe, wie Form, Muster oder Regel, sich als untauglich für die Beschreibung der uns begegnenden Phänomene erwiesen. Ein ,practice turn' in der Gestikforschung entsprach der Logik des Gegenstandes, so wie sie sich im Forschungsprozess allmählich zu erkennen gab: Erst als Realisierungen von Praktiken verstanden - und nicht als vorfindliche Formen und Formkombinationen - ergaben die beobachteten Phänomene für uns Sinn.

Im Folgenden stelle ich einige dieser Schritte von der Form zur Praktik dar, in einer Reihenfolge, die ungefähr dem Fortgang der Forschung entspricht. Das erste relevante Phänomen, das wir in allen unseren Videodaten entdeckten (vgl. Streeck 1988b, 1993, 2009: Kap. 4), war der ,Blick zur gestikulierenden Hand': Gelegentlich, aber offenbar mit Methode, schauten sich Sprecher für einen kurzen Augenblick auf die eigenen gestikulierenden Hände. Diese Beobachtung führte zur Beschreibung eines wiederkehrenden ,Musters‘, in dem Sprache, Geste und Blickverhalten miteinander koordiniert sind. Man kann dieses Muster einerseits als Konstruktion betrachten und dann sowohl Gemeinsamkeiten als auch Unterschiede in dieser Konstruktion zwischen den Sprachen feststellen. Betrachtet man diese Koordination demgegenüber als Praktik, würde man sie eher als ,sprachübergreifend‘, gleichsam als best practice ${ }^{1}$ für die Lösung der ubiquitären kommunikativen Aufgabe, sprachliche und gestische Beschreibungen synthetisieren zu müssen, verstehen und die sprachlichen Elemente und Formen als Ressourcen, ${ }^{2}$ deren Eigenschaften von Sprache zu Sprache variieren können. Es ist interessant, dass diese oft auch als Fokusmarker (ohne Geste; vgl. Wiese 2011; kritisch dazu Auer 2007, der in den meisten Fällen von einer Unschärfemarkierung ausgeht) verwendet werden, als gehörten die interaktive Fokussierung von Gesten und die sprachliche Fokussierung von new information auf natürliche Weise zusammen. Der Kontext dieser Fokussierung von Gesten ist ausnahmslos der des Beschreibens: Die Hände beschreiben ein Objekt oder ein Ereignis, in Ergänzung oder anstelle einer sprachlichen Beschreibung. Der Versuch, diese beschreibenden Gesten einer Formanalyse zu unterziehen, die der Formanalyse sprachlicher Einheiten analog ist, muss jedoch scheitern: Beschreibende Gesten (z.B. eines Sprechers) und ihre Bestandteile konstituieren kein Inventar aufzählbarer Formen, sondern sind ad hoc-Produkte, die prinzipiell, trotz zahlloser Ausnahmen, nicht je schon Besitz einer Gemeinschaft sind. Geteilt sind vielmehr

1 Die Åhnlichkeit oder Identität der Praktiken verschiedener Kulturgemeinschaften kann man als Resultat von Diffusion, mit größerer Wahrscheinlichkeit aber als Ergebnis paralleler Entwicklung erklären.

2 Resource im Sinne der Konversationsanalyse. 
Methoden des Beschreibens - Praktiken. Die Konstruktionen, die der Fokussierung der Geste dienen (und grammatisch beschreibbar sind), dienen also als Ressourcen für die Praxis gestischen (oder bimodalen) Beschreibens von Welt und sie sind vermutlich in hohem Maße auch ihr Produkt. Wie Verschiebungen kommunikativer Praktiken die Re-Analyse sprachlicher Einheiten bewirken, die zugleich durch sie ermöglicht werden, und wie der mimetischen Beschreibungen in Äußerungen Raum gegeben wird, werde ich abschließend an der Entstehung einer neuen sprachlichen Form zeigen, die eine spezifische Form körperlichen Ausdrucks, ,Körperzitate“ (Streeck 1988a), rahmt oder markiert, nämlich das verbum dicendi ,be like‘ im amerikanischen Englisch.

\section{Der Blick auf die gestikulierende Hand}

1987 habe ich an der FU Berlin zusammen mit Cornelia Müller, Ulrike Hartge und anderen Magisterstudenten begonnen, Handgesten in natürlichen kommunikativen Situationen in ,interaktionistischer Perspektive‘ zu erforschen. Unser Ziel war es, empirische Aussagen über die interaktiven und kommunikativen Funktionen spezifischer, bei der Materialsichtung zu identifiziender Formen von Handgesten zu machen, also zu klären, welche Beiträge diese Gesten nachweisbar zum kommunikativen Geschehen machen, wie sie von anderen Interaktanten aufgenommen werden, die sprachliche Äußerung ,kontextualisieren‘ oder ergänzen und mit ihr koordiniert werden. Als Konversationsanalytikern war uns klar, dass das ,doing ' einer Handgeste unauflöslich mit dem Augenblick verschmolzen ist, an der sie ausgeführt wird, und deshalb achteten wir bei unseren Transkriptionen und Analysen sehr genau auf das ,micro-timing von Handbewegung und Wort. Schegloff (1984) hatte in dem einzigen Artikel zu nichtsprachlichem Handeln, der in der ersten Generation von Konversationsanalyse erschienen war, Beobachtungen von Kendon (1980) und anderen aufgreifend das ,pre-positioning von Gesten im Verhältnis zu ihren ,lexical affiliates' beobachtet und Gesten zutreffend als ,projection device“ beschrieben, d.h. als Verhaltenseinheit, die andere Verhaltenseinheiten gleichsam vorankündigt. ${ }^{3}$ Damit schien für uns eine klare Forschungsstrategie vorgezeichnet, die gleichsam die strukturalistische FormBedeutungs-Analyse konversationsanalytisch flektierte: Es galt, die interaktiven Bedeutungen von gestischen Formen zu erfassen, die an spezifischen ,Turn-Posi-

3 Im Laufe der Zeit zeigte sich allerdings, dass dies nur eine mögliche ,Stellung‘ von Handgesten in ,turns-at-talk' ist. 
tionen (vor Turn-Beginn, am Turn-Beginn, vor Turn-Ende, im ,transition space, usw.) sowie an spezifischen syntaktischen Positionen ausgeführt werden. Auf diese Weise würden Gesten als ,conversational objects‘ verstehbar werden, d.h. als identifizierbare Verhaltenseinheiten, deren Existenz sich spezifischen Kommunikations- oder Strukturierungserfordernissen verdankt.

Ungeachtet dieses planvollen Forschungsvorhabens war das erste ,Phänomen', das sich uns zeigte, unerwartet und anderer Natur und eher eine Begleiterscheinung des Gestikulierens als im engeren Sinne ein gestisches Phänomen: In unseren Daten - Videoaufnahmen von nicht vorstrukturierten Gesprächen unter Freundespaaren - fiel auf, dass sich Sprecher, wenn sie gestikulieren, ,immer wieder einmal' auf die eigenen, gestikulierenden Hände schauen. Dies geschieht mehrfach im folgenden Datenausschnitt; der Blick auf die eigene Hand ist mit einer Schlangenlinie in der Transkriptzeile oberhalb des Redetranskripts markiert. Eckige Klammern markieren die (Dauer der) Handgesten, die gleichzeitig ausgeführt werden. Nur solche Aspekte des Kommunikationsprozesses, die hier unmittelbar relevant sind, werden in den Transkripten dargestellt. In diesem Ausschnitt erzählt Christine ihrer Freundin Simone von einer Aufführung von Kleists „Penthesilea“ in einer Inszenierung von Robert Wilson. (Ich sehe hier davon ab, die Form der Gesten zu beschreiben und setze die Eindeutigkeit ihrer Referenzen voraus; zu den Beschreibungsmethoden vgl. Streeck 2009, Kap. 6.)

01 C: Dritte Szene wa:r ohne Worte. 


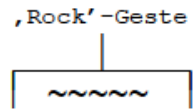

In diesem Ausschnitt schaut die Sprecherin dreimal auf ihre gestikulierende Hand: siehe Zeilen 05, 06/07 und 12. In Zeile 05 hebt die Sprecherin die linke Hand und greift mit ihr eine Form, während sie ,hatten se so' sagt, und bei ,so' fällt ihr Blick auf ihre Hand, um dann mit dem nächsten Wort, ,Fetzen‘, dem ,lexical affiliate“ (Schegloff 1984) der Geste, zu Simone zurückzukehren. Mit ihrer nächsten Geste zeigt sie das ,Format‘ der Fotos: Beide Hände zeigen gemeinsam nacheinander Höhe und Weite. Der Blick der Sprecherin ist auch hier auf ihre Hand fokussiert, als sie ,so' sagt. Schließlich wiederholt sich das Schema in Zeile 12, wo Christine sich mit der Hand über den Bauch fährt, den Saum eines Rockes suggerierend, und zugleich mit den Augen gleichsam auf die Geste ,zeigt‘.

In (2) spricht Dirk, ein junger Arzt, von den Schwierigkeiten der Arbeitssuche. Er erzählt, wie er auf dem Arbeitsamt war und dort ein Merkblatt in der Hand hielt. Dabei hat er Schwierigkeiten, das Wort ,Merkblatt‘ $\mathrm{zu}$ finden und wiederholt stakkato-artig ,son', bis er die Hände zu einer Geste hebt und gleichzeitig den Blick den Händen zuwendet. Die Hände begegnen sich und sind nach oben hin offen - wie eine Broschüre oder ein Buch, das man liest, oder ein Faltblatt. Dirks Blick auf seine Hand ,zeigt“ auf seine Geste, ist zugleich aber auch Teil der Darstellung: Dirk stellt sich selbst merkblattlesend dar.

01 Dirk Also ich hatte- ich hatte letztens w- irgendwo a äh äh ähm aufm Arbeitsamt? oder (.) was das war.

Dieser ,Blick zur Hand‘ seitens des gestikulierenden Sprechers, nicht irgendeine Geste oder ein Stellungsmerkmal von Gesten, wurde so zu unserem ersten ,Phänomen' oder ,conversational object'. Es handelt sich bei diesem Phänomen nicht allein um den Blick zur gestikulierenden Hand, sondern um ein dreigliedriges 
Objekt, bestehend aus einer sprachlichen Einheit und zwei Einheiten körperlichen Handelns: In jedem Falle sagten die Sprecher ,so‘, während sie ihren Blick ihrer Geste zuwandten. Man könnte dieses konversationelle Objekt als eine multimodale Konstruktion im Sinne der Konstruktionsgrammatik (vgl. Zima 2014) beschreiben: Demnach gehörte zu den grammatischen Konstruktionen des Deutschen eine, die ein deiktisches Adverb, ,so‘, mit einer simultanen Handgeste und einem simultanen vorübergehenden Blickwechsel zur Hand verbindet und einem verbalen, nominalen, adjektivischen oder präpositionalen Deskriptor vorgeschaltet ist: ,son^ ${ }^{\wedge}$ Geste ${ }^{\wedge}$ Blick zur Hand + NP/VP/ADJ/ADV“ (,son Merkblatt‘, ,so in diesem Format' etc.). Und man könnte nach weiteren multimodalen Konstruktionen suchen, d.h. Konstruktionen, die sprachliche (akustische) und gestische (visuelle) Einheiten miteinander verschweißen und dazu dienen, die Aufmerksamkeit des Hörers auf die Geste zu lenken, diese also ,relevant zu setzen“ (Kallmeyer/Schmitt 1996). Um diese Konstruktionen adäquat zu beschreiben und um die Verstehbarkeit eines Satzes wie ,hatten so Fotos, so in diesem Format' zu erklären, ist es unabdingbar, das ,nichtverbale“ Element in die Beschreibung aufzunehmen: Ohne eine Handgeste ist dieser Satz ungrammatisch (und unverstehbar). Sprachimmanente Grammatik stößt hier an ihre Grenzen.

Doch wäre mit der grammatischen Beschreibung dieser trimodalen Konstruktion und ihrer Aufnahme in die Grammatik des Deutschen noch nicht viel gewonnen, denn diese Beschreibung sagt uns ja nicht, was die Sprecherin, die eine solche Konstruktion realisiert, damit bewerkstelligt. Die Antwort auf die Frage nach dem doing des ,Phänomens“ erscheint im Nachhinein einfach, fast trivial, doch zeigte sie sich uns im Verlaufe von mühsamer und langwieriger Kontextanalyse (vgl. Scheflen 1974): Die Sprecher beschreiben in allen Fällen ein Stück gelebte, wahrgenommene oder imaginierte Realität, und sie tun dies unter anderem oder ausschließlich mit den Händen. Die Konstruktion ist Teil von Beschreibungspraxis und zugleich Teil des deiktischen Systems des Deutschen (siehe Stukenbrock 2010, 2015 und in diesem Band). Im Forschungsprozess selbst zeigte sich zunächst nur der Blick zur Hand, ohne dass man ihm ansehen konnte, auf welchen Kontext er bezogen ist und was mit ihm bewerkstelligt wird. ${ }^{4}$ Deutlich war, dass Sprecher nur gelegentlich ihren Blick ihren Gesten schenken; der weitaus größte Teil aller konversationellen Gesten bleibt unbeachtet, nichts deutet darauf hin, dass

4 Erstaunlicherweise scheint das ubiquitäre Phänomen des Blicks zur gestikulierenden Hand uns als Alltagsmenschen vollkommen unbekannt zu sein; niemand, mit dem wir sprachen, war sich dieses Verhaltens bewusst, doch hatte nachträglich dann auch niemand Schwierigkeiten, ihn zu beobachten. Auch in der Literatur, mit Ausnahme von Goodwin (1986), wurde er bis dato nirgends beschrieben. 
die Teilnehmer auf die Gesten achten, und noch weniger, dass ihre Produzenten darauf achten, dass auf sie geachtet wird. Dies ist aber regelmäßig bei beschreibenden Gesten der Fall (Streeck 2008): Hier gestikulieren Sprecher nicht nur, sondern sie stellen durch die Positionierung der Geste und ihr begleitendes Verhalten sicher, dass die Geste zur Kenntnis genommen wird. Der Zusammenhang mit Beschreibung wurde erst allmählich deutlich, durch die andauernde Reflektion darauf, was die Geste in ihrem Kontext ,tut', beziehungsweise welcher Art der Kontext ist, auf den sie sich bezieht (vgl. Streeck 2009). Der Blick zur Hand ist regelmäßig Teil einer trimodalen Konstruktion, und diese wird stets im Kontext von Beschreibung realisiert und hat die Funktion, die Aufmerksamkeit des Hörers auf die gleichzeitige Handgeste zu richten, sie also vom ,verbalen zum visuellen Kanal` umzulenken; nur so wird die gegenwärtige sprachliche Äußerung verständlich. Reflektiert man zugleich die verschiedenen Lesarten von ,so' und ihre größere und geringere ,Wörtlichkeit', so erscheint dieser Gebrauch von ,so - in Verbindung mit einer beschreibenden Geste - ein prototypischer Gebrauch zu sein: Adverbiales ,so“ (vgl. Auer 2007; Ehlich 1986) bedeutet, dass eine Art und Weise nicht benannt wird, sondern gegenwärtig irgendwo angeschaut werden kann, nämlich dort, worauf ,so' verweist, bzw. wofür ,so', um eindeutig zu verweisen, noch einen ,Pfeil“ oder ,Wegweiser ' braucht, den es im Wechsel der Blickrichtung, also im Hinschauen-auf, findet. Wenn der Blick auf die Umgebung verweist, vervollständigt diese (bzw. ein Objekt in ihr) die Bedeutung, und wenn der Blick dabei auf die Hände des Sprechers fällt, dann ist es die Geste, die diese Vervollständigung leistet.

Mit dieser Zuordnung zu einem Handlungskontext haben wir den Blick zur Hand als Teil einer Praxis identifiziert. Wir explizieren die Form, indem wir sie einer Klasse von Handlungen zuordnen. Dass unsere Lesart dieser Form zutreffend war, zeigte sich vor allem an zwei weiteren Befunden: Einerseits den Reaktionen der Hörer in unseren und anderen Daten, zum anderen dem Auftauchen von Phänomenen, die der ,so+Blick'-Konstruktion hochgradig ähneln, in unseren fremdsprachigen Daten. Zum ersten: Wir konnten zeigen, dass Rezipienten einer Äußerung, die diese Konstruktion enthält, ihrerseits ihren Blick der gestikulierenden Hand des Sprechers zuwenden (Streeck 1994). Sie ,ratifizieren‘ mit anderen Worten mit ihrem eigenen Blickwechsel den Blickwechsel und zugleich die Geste des Sprechers. Gullberg hat diese Befunde in experimentellen Untersuchungen mithilfe von ,eye tracker'-Technologie bestätigt und zugleich überzeugend argumentiert, dass es sich bei diesen Blickwechseln weniger um einen für das Verstehen notwendigen ,kognitiven“ Wahrnehmungsprozess handelt, sondern eher um soziale Kundgaben und Ratifizierungen: Mit dem ,return gaze‘ zur gestikulierenden Hand affirmiert die Zuhörerin, dass sie die Geste zur Kenntnis nimmt, wobei aber die ,kognitive‘ Kenntnisnahme der Geste dieses Blickwechsels eigent- 
lich nicht bedarf, da sie ohnehin im Blickfeld der Zuhörerin vollzogen wird (aber eben nicht des Sprechers selbst). Der Hörerblick zur Hand ist ein Akt sozialer Anerkennung (Gullberg/Holmqvist 1999; vgl. Streeck 2014).

Aus forschungsstrategischen Gründen erhoben wir für unsere frühen Gestikstudien ein mehrsprachiges Datenkorpus und analysierten die uns sprachlich nicht zugänglichen Videoaufzeichnungen (u.a. von Gesprächen in Japanisch, Thai und der philippinischen Sprache Ilokano) im Hinblick auf auffällige Phänomene körperlicher Interaktion und Kommunikation, bevor wir die von Muttersprachlern gefertigten Transkripte und Übersetzungen sowie ihre Intuitionen zu Rate zogen. Unser Bemühen war, uns Gesten über ihre Formen, nicht über sprachlich kommunizierte Bedeutungen zu nähern. Blicke zur Hand fanden wir in allen Gesprächen, auch solchen zwischen Mitgliedern asiatischer Kulturen, denen oft ein ganz anderes Blickverhalten nachgesagt wird. Wie weit Blickverhalten zwischen Kulturen differiert, ist ungewiss. Dass ,so' ein Wort einer einzelnen Sprache ist, ein deutsches Wort, ist unbestritten, und wir konnten deshalb kaum mit dem Auftauchen direkt übersetzbarer Gesten-Fokussierungskonstruktionen in anderen, zumal typologisch so unähnlichen Sprachen wie dem Japanischen, Thai und Ilokano (Philippinen) rechnen. Wie sehr sich die Phänomene dennoch ähneln, zeigt Beispiel (3), aus einem Gespräch, in dem zwei Japanerinnen in Berlin, nachdem sie über die Schwierigkeit, hierzulande einen Führerschein zu erwerben, gesprochen haben, einander von Autounfällen erzählen, in die sie in Japan verwickelt waren. Hier sehen wir eine kanonische Konstruktion, die in verschiedenen Varianten zu Dutzenden Malen in diesem siebenminütigen, beschreibungsreichen Gesprächsausschnitt vorkommt.

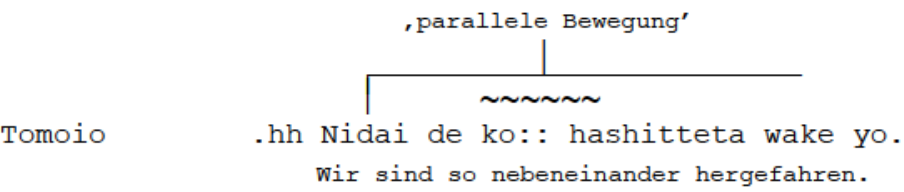

Die Sprecherin zeigt mit einer bilateralen Geste, wie zwei Autos nebeneinander her fuhren, und schaut sich dabei kurz auf ihre Hände, zur gleichen Zeit, als sie ,ko äußert, eine demonstratives Adverb, das man zweifelsohne mit ,so“ übersetzen muss, unbeschadet möglicher Verschiedenheiten in den syntaktischen Restriktionen (Stellungsbeschränkungen) der zwei Einheiten. In Ausschnitt (4) beschreibt die andere Teilnehmerin, Satomi, wie sie ihrerseits in einen Unfall geriet und versuchte, den Wagen in der Spur zu halten, aber die Kontrolle verlor, und dabei verbindet sie wiederholt Geste, Blick zur Hand und ,ko. 
(4)

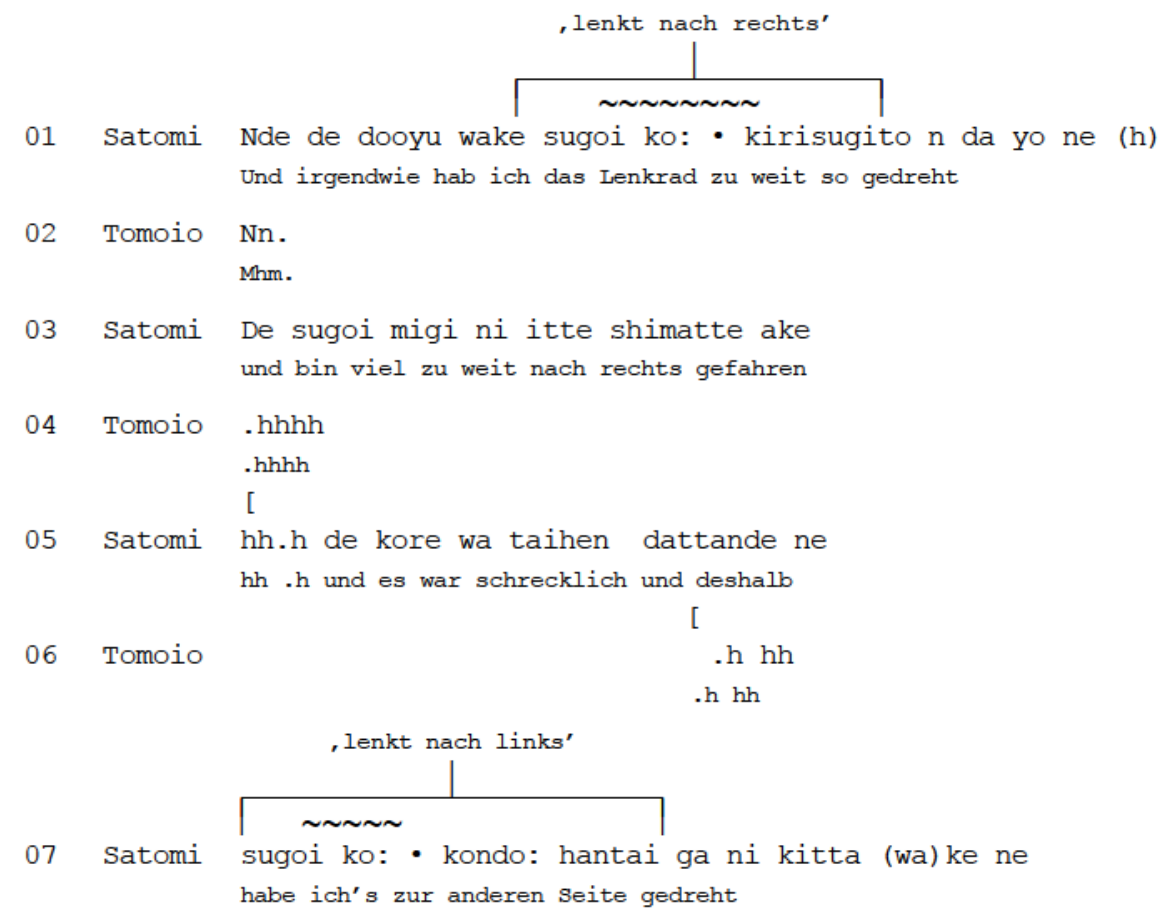

Wir sehen, dass die japanischen Sprecherinnen im Wesentlichen die gleiche Praktik - bzw. die gleiche Kombination von Praktiken - realisieren wie die deutsche. Wir müssen offenlassen, wie weit die syntaktische Charakteristik von ,ko' (seine Kombinierbarkeit) derjenigen von ,so' gleicht. ,So kann immerhin mit dem unbestimmten Artikel fusionieren (vgl. von Heusinger 2012; Hole/Klumpp 2000; Knöbl 2014): ,so ein', ,son', ,sone‘ etc., wie im Beispiel (2), das heißt, es kann auch bei der gestischen Beschreibung von Dingen eingesetzt werden und steht damit letztlich für fast alle gestischen Beschreibungskontexte zur Verfügung. Ob dies auch für ,ko‘ gilt, wäre zu klären. Man kann aber mit guten Gründen annehmen, dass das gestische Beschreiben - die Möglichkeit, beschreibende Gesten als grammatische Äußerungsbestandteile zu integrieren (deren Beachtung durch den Hörer damit einklagbar wird) - ein prototypischer, originärer Verwendungskontext von ,so' und ,ko' ist, von dem ,bleichere“ Verwendungen (z.B. als Fokus-Marker, Gliederungssignale etc.) abgeleitet sind. Im Englischen steht kein Äquivalent von, so zur Verfügung. Im Kontext von Nominalphrasen wird die Verknüpfung zur Geste mit dem Demonstrativum ,this“ hergestellt, das freilich nicht in Verbalphrasen verwendet werden kann. Dort kann man ,like this‘ sagen (,and then he walked 
like this'), doch ist dies dem Verb nachgestellt und hat damit nicht jene projektive Wirkung, auf die beginnende Geste aufmerksam zu machen, die ,so‘, verbunden mit dem Wechsel der Blickrichtung, innewohnt.

In anderen Sprachen gibt es andere Mittel der grammatischen Integration von Gesten, in der philippinischen Sprache Ilokano ${ }^{5}$ zum Beispiel das demonstrative Adverb ,kastoy“ - ,like this‘, , auf diese Weise“ -, das auch idiomatisch als finale Satzpartikel verwendet werden kann (,so ist es'), interessanterweise nicht selten nach Äußerungen, die von einer Geste begleitet waren. ,Kastoy“ kann aber auch durch Affigierung selbst zu einem Verb gemacht werden (,ich habe so gemacht', ,ich habe gedingst'), wie in Beispiel (5). Hier sprechen drei Frauen über traditionelle Heilmethoden, gegenwärtig die, ein krankes Kind über einem Feuer hin und her zu schwenken, um es zu wärmen. Eine Frau hat die Methode beschrieben, und eine andere fragt ungläubig nach, wobei sie die Arme mit dem vorgestellten Kind im Interaktionsraum ausgestreckt hin und her bewegt: ,du hast es gesost?‘. Die Verbform ist ,i-kas-kastoy-mo-n‘: ABL-ITV-so-2.P.Sg.-PFT. Die iterative Form drückt das wiederholte Hin und Her der Bewegung aus und ist ,ikonisch“ durch Reduplikation der ersten geschlossenen Silbe von ,kastoy (,kas-kastoy`) markiert. Das Ablativ-Präfix ,i` korrespondiert den ausgestreckten Armen: Das Baby wird vom Körper weg gehalten. Der Bezug zur Geste ist also tief in die grammatisch-konzeptuelle Struktur der Verbalphrase eingebettet.

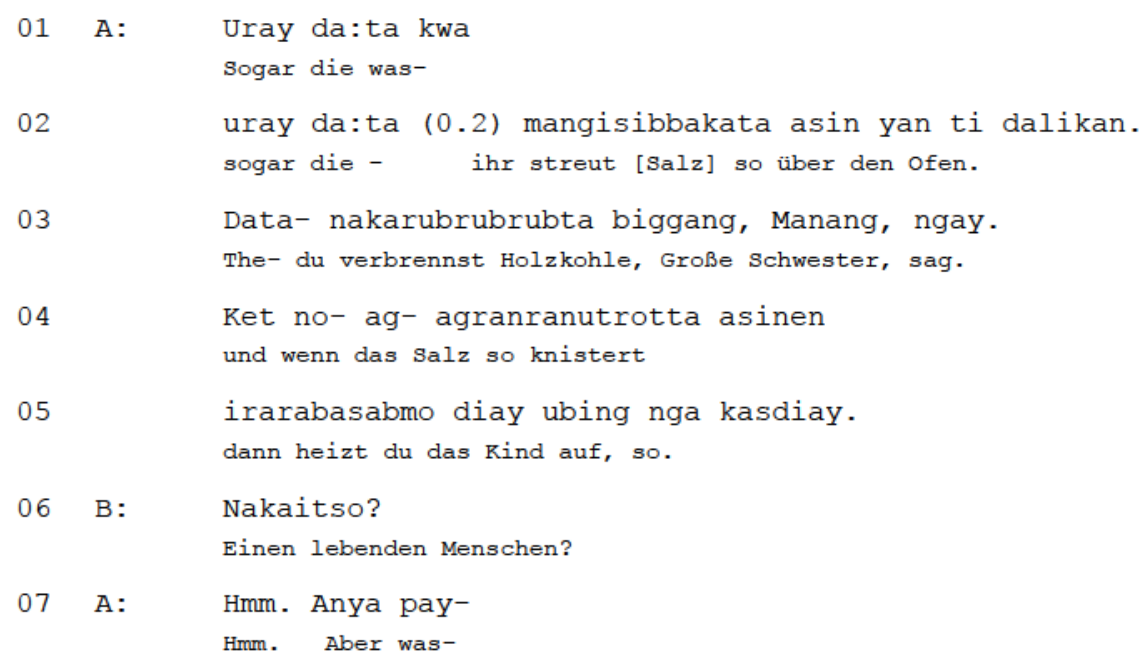

5 Das Projekt über Ilokano, „Sprachtypologie und Interaktion“, wurde von der Deutschen Forschungsgemeinschaft gefördert. 


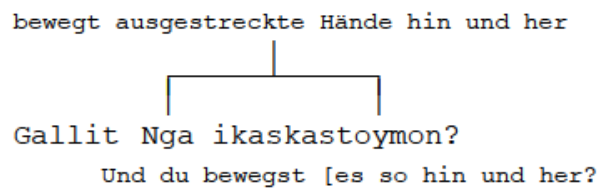

In Ilokano-Gesprächen kann man auch eine andere Praktik beobachten, die in ähnlichen Kontexten auch im Deutschen (vgl. Beispiel (2)) und vermutlich zahllosen anderen Sprachen realisiert wird, nämlich ein Funktionswort oder Morphem stakkatoartig zu wiederholen, wenn nach einem Wort gesucht wird, und dabei mit der Hand beschreibend zu gestikulieren. In Beispiel (6) ist dies das Portmanteau-Präfix ,in', das sowohl Vergangenheit wie ,undergoer focus' ausdrückt (d.h. der Satz handelt vom direkten Objekt des Verbs). Man könnte diese Wiederholung im Deutschen als ,hatte hatte hatte' oder ,wurde wurde wurde‘ wiedergeben. Vor dieser Konstruktion erscheint jedoch schon das ,Wortsuchenwort' ,kwa', das in Wörterbüchern mit ,whatchamacallit‘ übersetzt wird und ebenfalls affigierbar ist: ,in-kwak in in in', sagt die Sprecherin, ,ich was-hatte hatte hatte“, und bewegt dabei Zeigefinger und Daumen einer Hand im Interaktionsraum: Mit ,ich hatte das Telefon leiser gestellt' vervollständigt sie später den Satz. (Ich werde auf ,kwa‘ noch einmal zurückkommen.)

A Inkabilko ti telepono idia kwa: - iday sa:la, kunak a. Ich hatte das Telefon in das was gestellt, ins wohnzimmer, hab ich gesagt. Zeige- und Mittelfinger auf und ab

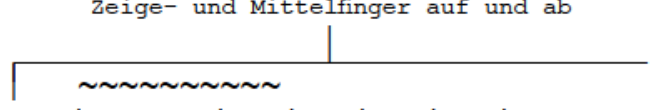

Sa inkwak- in- in- in- in- in- uhhund ich hatte es was (es es es es es ${ }^{* 6}$ ) äh-

6 ,Es‘ gibt die Bedeutung von ,in“ nur unzureichend wieder: ,in‘ ist die Vergangenheitsform des ,undergoer focus'-Präfixes, bedeutet also ungefähr ,es ge-' (wie in ,es gestellt'), aber niemand würde bei einer Wortsuche ,es ge-' wiederholen. 


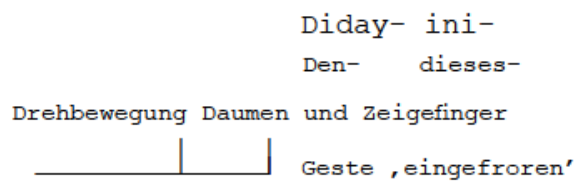

06

Haan! Didiay- ini::::: sangagd ngay:: . Nee! Den- diesen Knopf sag.

Hnn.

Hnn

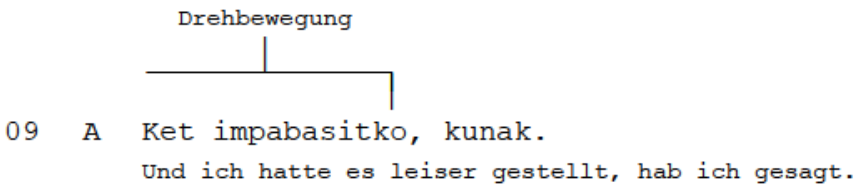

Wie im deutschen Beispiel (2) die Wiederholung von ,son', markiert die Wiederholung von ,in“ im Ilokano, ebenso wie ,kwa‘, dass eine Suche stattfindet, zeigt jedoch nichts über den Inhalt des gesuchten Wortes, nur eine Auswahl seiner grammatischen Bestimmungen, nämlich in Ilokano, dass es ein Verb im ,undergoer focus ' in der Vergangenheit ist, im Deutschen (,son'), dass es ein maskulines Nomen im Singular ist. Das Konzept - der ,Wortinhalt ${ }^{*}$ wird gestisch in Szene gesetzt.

Sofern man bereit ist, die systematische semantische und syntaktische Inkorporierung von Handgesten in die Form sprachlicher Äußerungen selbst als Teil von Grammatik zu betrachten, kann man ohne Weiteres diese multimodalen Fabrikationen - Subj.+V+son+Geste+NP bzw. UF+kastoy+Subj und UF-kwa-Subj - als Konstruktionen des Deutschen bzw. des Ilokano betrachten. Beide Sprachen - und man darf vermuten: alle Sprachen - kennen offenbar bimodale bzw. trimodale Konstruktionen, die Wörter und Gesten so zusammenschweißen, dass die Geste als semantisches Äquivalent eines (noch fehlenden) Wortes bzw. als visueller Äußerungsbestandteil erscheint. Die Konstruktionen gleichen sich insoweit, als sie auf bimodale Beschreibungsaufgaben zugeschnitten sind und diese routinemäßig lösen. Sie unterscheiden sich, insofern die für sie benutzten Wörter (,so‘, ,kastoy‘, ,this‘ etc.) unterschiedlichen Restriktionen ihrer Stellung im Satz unterliegen. (Andere Faktoren mögen hinzutreten.) Aber ebenso gut kann man diese Konstruktionen auch als Praktiken verstehen, da sie ja eine Methode für die koordinierte, dynamische, situative Realisierung verschiedener Ressourcen darstellen; sie wären insofern Elemente einer ,grammar of use oder ,grammar of action' (wobei hier der Begriff ,Grammatik` eher metaphorisch gebraucht 
wird). Der Unterschied ist lediglich einer des Blickwinkels, ob man die multimodale Form von ihrem Abschluss her - als hergestellte Konstruktion - oder von ihrem Vollzug her - als Handlungs- und Kommunikationsmethode - betrachtet (die sprachabhängig variiert). Entscheidend für uns ist die Interdependenz von Form und Praktik: Die geronnene sprachliche Form verdankt sich der kommunikativen Praktik, deren Ressource sie zugleich ist. Und sobald sie in einer Gemeinschaft habitualisiert ist, wird sie auch für andere Praktiken verfügbar, ,kwa' zum Beispiel, ebenso wie ,son/sone‘, als Fokusmarkierung.

Im Beispiel (7) markiert ein Landbesitzer auf den Philippinen bei einer BibelExegese für seine Arbeiter eine Konstituente nach der anderen, indem er sie als Suche konstruiert, wodurch er ebenso gravitätisch erscheint wie seine unvollständige Sprachkenntnis preisgibt (seine Muttersprache ist Tagalog).

(7) Venturina

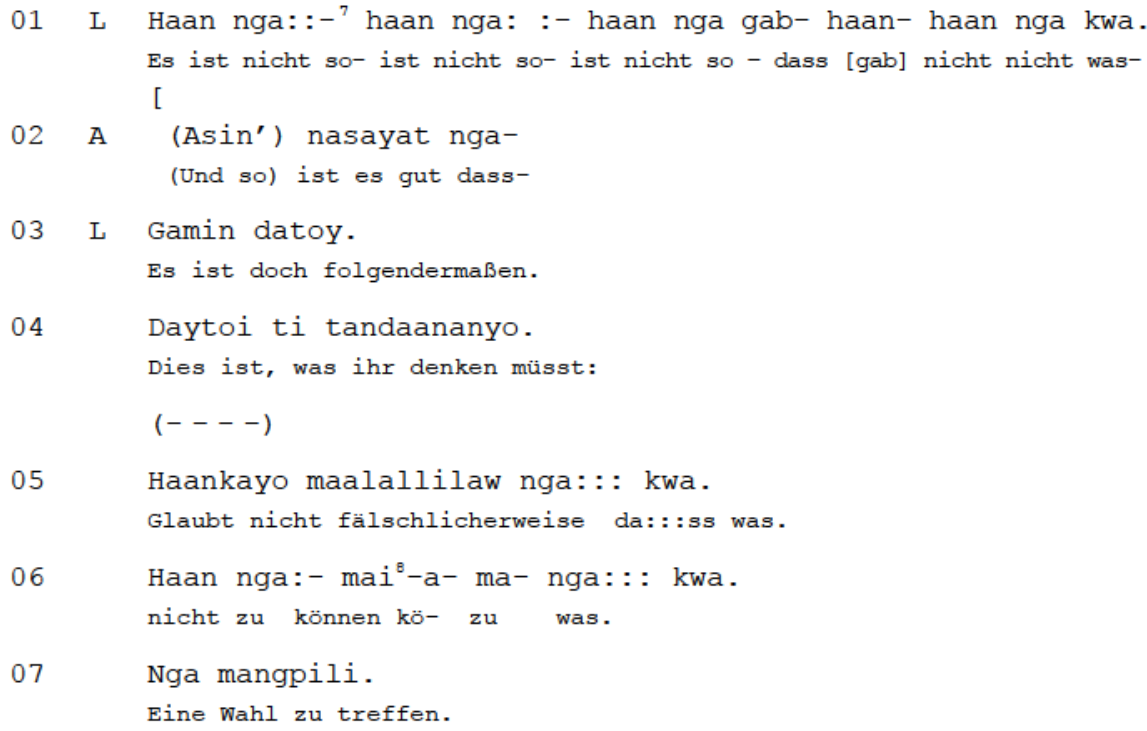

Dies Datum zeigt ,kwa‘ auf zwei verschiedenen Grammatikalisierungsstufen zugleich, angesiedelt an der Schnittstelle zwischen zwei Praktiken, Wortsuche und Fokussierung.

7 ,Haan' ist ein ,Existenzverb‘, dass der negierten Proposition vorausgeht und mit ihr durch die Ligatur ,nga' verbunden ist. Die Bedeutung von ,haan nga' ist also ,es ist nicht der Fall, dass'.

8 Das Präfix ,mai-‘ drückt im Wesentlichen die Fähigkeit etwas zu tun aus. 


\section{Praktiken gestischer Beschreibung}

Setzt man diese Untersuchung der Formen und Funktionen von Handgesten fort, indem man neben ihrer Einbettung in sprachlich-grammatische Einbettung auch die Form der beschreibenden Gesten selbst in Augenschein nimmt, muss man allerdings die strukturale, von der sprachlichen Form aus vorgehende Analyseweise bald zugunsten einer praxeologischen aufgeben. Denn anders als die Wörter, die sie ergänzen oder ersetzen, sind beschreibende Gesten in keinem geteilten Lexikon enthalten, sie sind, von Ausnahmen abgesehen, nicht aufzählbar wie die Wörter einer Sprache. Sie sind vielmehr überwiegend improvisierte, situative ,Erfindungen“ (vgl. Donald 1991). Als solche können sie auch fehlschlagen. Im Beispiel (6) z.B. wird die ,Leiserstell'-Geste der Sprecherin nicht verstanden, deshalb modifiziert, und wieder nicht verstanden. Die Sprecherin findet das Wort ,pa-basit" (CAUS-klein) schließlich selbst. Die ad hoc-Natur gestischer Beschreibung wird durch den Begriff der ikonischen Geste, der in diesem Zusammenhang in der Regel benutzt wird, verschleiert, weil er suggeriert, dass es so etwas wie ein Vokabular ,fertiger' ikonischer Gesten gibt. Zwar gibt es wohl in allen Kommunikationsgemeinschaften emblematisierte (konventionelle) ikonische Gesten wie etwa die für Trinken, Telefonieren und etliches mehr, doch geht es oft dann, wenn gestisch beschrieben wird, darum, Details zu illustrieren, die sprachlich nur schwer kodierbar sind. Die obigen Wortsuchen sind in dieser Hinsicht schlechte Beispiele, da in ihrem hochspezifizierten Kontext in der Regel eine einzelne Geste ausreicht. Komplexere Darstellungsaufgaben erfordern komplexere, diversifizierte und kombinierbare Darstellungen.

Es ist also die Geläufigkeit der Darstellungsmethoden, nicht der einzelnen Form, die das Gelingen von gestischen Beschreibungen ermöglicht. Wenn man sich mit kommunikativen Praktiken des Körpers befasst, hat man es zunächst nicht mit festen Formen oder Form-Bedeutungs-Beziehungen zu tun, sondern mit Methoden der Bedeutungserzeugung. Konventionelle ikonische Gesten geben ein schlechtes Modell für gestische Verständigung im Kontext von Beschreibungen ab. Für Objekte wie etwa Autoreifen und Absperrgatter gibt es keine gestischen ,Namen', so wie Nomina ,Namen' von Dingen sind. Objekte dieser Art (komplex, nicht hand-gerecht, u.U. selten) müssen irgendwie nachvollziehbar ,evoziert', ,insinuiert' werden. Dies kann nur gelingen, wenn es einen common ground von Beschreibungsmethoden und eine gemeinsam bewohnte Welt gibt. Dass wir über gestische Beschreibungen noch nicht so viel wissen, wie wir könnten, liegt vor allem daran, dass Ikonizität in der Semiotik in der Regel als eine Sache der Ähnlichkeit zwischen Zeichen und Referent erklärt und damit zu einem Nicht-Problem gemacht wird (Ähnlichkeit ist ja eine ,natürliche', nicht weiter erklärungsbedürftige Beziehung), doch lehrt die Empirie, dass die Beziehungen vielfältiger 
sind, und es gibt darüber hinaus auch logische Einwände gegen diese Erklärung (Goodman 1976; siehe Streeck 2008). Was ,ikonische‘ Gesten anbetrifft, so charakterisieren diese Objekte und Ereignisse auf ,handgerechte‘ Art, d.h. in einer Weise, die oft mit den präsymbolischen Objektkontakten und Welterfahrungen menschlicher Hände zu tun hat. Als Tomoio bei ihrer Unfallschilderung beschreibt, dass auf regennasser Straße die Räder ihres Wagens durchdrehten, nimmt sie zum Beispiel sichtbar einen kleinen Reifen in die Hand und dreht ihn nach rechts und links: In der wirklichen Welt könnte sie dies nicht (sie bräuchte gewiss zwei Hände, andere Griffhaltungen und Bewegungen), das Rad ist radikal verkleinert, aber ein rundes, rotierendes Objekt, zum Beispiel das Rad eines Spielzeugautos - kennt eine menschliche Hand eben als ein Ding, das sie drehen kann. Vom Größenunterschied und den unterschiedlichen affordances der zwei Arten von Rädern abstrahiert die Geste, so wie alle beschreibenden Gesten ,verallgemeinern“ (vgl. Arnheim 1969). Das Schutzgatter, gegen dessen Ende Tomoios Auto prallte, wird von ihr in einer Wortsuche mit einer gekrümmten Hand dargestellt, die auf und ab fährt. Wir hatten die Geste zunächst für eine Pfostengeste gehalten, doch machte dies im Zusammenhang des beschriebenen Geschehens keinen Sinn. Erst eine Ortsbesichtigung japanischer Landstraßen durch einen heimreisenden Studenten ergab die Lösung, dass die Geste offenbar das gekrümmte Ende einer horizontalen Strebe darstellen sollte. Dies zeigt, dass beschreibende Gesten immer einen geteilten Welthorizont voraussetzen, eine den Körpern gemeinsam vertraute Welt. Erst diese macht die Gesten lesbar.

Ich habe in meinen empirischen Studien zur Gestik (und im Austausch mit Müller und Kendon; vgl. Müller 1998, Kendon 2004) in einem halben Dutzend von Sprachgemeinschaften zwölf verschiedene Darstellungspraktiken ausmachen können; zweifelsohne werden sich weitere finden und wird die Heuristik revidiert werden müssen (für detailreiche Beispiele siehe Streeck 2008 und 2009, Kap. 6). Hierzu gehören unter anderen (in englischer Terminologie):

- modeling: Die Hand wird als Repräsentant eines Objekts benutzt (vgl. Kendon 2004);

- bounding (vgl. Sowa 2007): Durch ihre Positionierung zeigen Daumen und Zeigefinger oder die zwei Hände das Maß eines Objekts;

- drawing: Der Zeigefinger produziert eine Umrisszeichnung im Interaktionsraum;

- handling: Die Hand oder die Hände evozieren die Vorstellung eines Objekts durch eine ,typische Bewegung;, d.h. eine schematische Handlung, die mit dem Objekt routinemäßig ausgeführt wird; diese charakteristischen Handlungen variieren in ihrer Spezifizität, von generischen ,Transporthandlungen ( aufheben, niedersetzen, halten) bis zu Bewegungen, die eine bestimmte Objektgattung eindeutig identifizieren; 
- making: Die Hände schaffen ein (virtuelles) Objekt, setzen es zusammen oder formen es wie aus Ton (molding);

- scaping (ein Kunstwort): Die Hände formen eine virtuelle Landschaft durch Akte, die denen ähneln, mit denen man Spiellandschaften am Strand formt;

- acting: Die gestische Handlung dient als abstrakte Version einer realen Handlung (um die Handlung, nicht - wie bei handling - ihr Objekt zu evozieren); und

- pantomime: Das Verhalten von Figuren wird, im Kontext von Erzählungen und direkter Rede, ,re-animiert‘ (Goffman 1981).

Vergleicht man diese Praktiken, gewinnt man den Eindruck, dass einige an andere Kulturtechniken, drawing zum Beispiel an das Zeichnen in Staub oder Sand gebunden sein könnten, denn nur vor einem solchen materialen Hintergrund sind ,Luftzeichnungen` vermutlich verstehbar. Andere Praktiken werden offenbar im direkteren Rückgriff auf das konkrete Körperwissen des jeweiligen Leibes realisiert, etwa, wenn ein Arbeiter die Bedienung einer Maschine erklärt. Betrachtet man längere gestische Erzählungen, so sieht man, dass Sprecher fortlaufend die Darstellungsmethode wechseln, ohne dass Hörer damit Schwierigkeiten zu haben scheinen. Die Japanerinnen aus den obigen Beispielen wechseln vom scaping des Terrains zum ,thetischen' Hinstellen eines Baumstumpfes, zu Handbewegungen, die das Schlingern des Autos modellieren, zu einer mimetischen Re-Inszenierung des Aufpralls ,in der ersten Person“ (der Oberkörper wird nach vorn geworfen). Ein Kunsthistoriker in meinen Videodaten animiert pantomimisch, detailliert und mit großer Sachkenntnis die vielfältigen Produktionstechniken von Jackson Pollock, um dann einen Farbeimer abstraktiv mit einer angedeuteten, zweihändigen Bewegung vor dem Hörer ,abzustellen'. Bei aller Vielfalt scheint der default-Modus gestischen Beschreibens, realisiert vor allem bei beiläufigen Beschreibungen, der des handling zu sein, vermutlich, weil hier die Hände ihren Referenzobjekten gewissermaßen am nächsten sind, weil sie sich auf ihre eigenen Handlungsgewohnheiten und routinierte Weltkenntnis verlassen können. Besser gesagt vertraut der Sprecher auf die autonomen Handlungsfähigkeiten seines Körpers. Dies ist bei anderen, weniger fokalen Formen des Gestikulierens noch mehr der Fall (z.B. wenn Sprecher ihre Sprechakte unbewusst durch metaphorische Handlungen wie Greifen, Beiseiterücken, Teilen u.Ä. in Szene setzen). Wie welche Sprecher welche Darstellungsroutinen habitualisieren und wie sie diese in Situationen realisieren und fortentwickeln, darüber wissen wir bisher fast nichts (obwohl die Beschreibungspraktiken einzelner Berufsgruppen beschrieben worden sind; vgl. insbesondere Sauer 2003).

Beschreibende Handgesten lassen sich also nicht grammatisch beschreiben, insofern man unter Grammatik die Beschreibung von Formen und ihren Bedeu- 
tungen und Vorkommen in Konstruktionen und Ausdrücken versteht. Was stattdessen möglich ist, ist eine Praxeologie gestischer Beschreibung, das heißt ein beständiges aufmerksames Sammeln von gestischen Darstellungsmethoden, und die Analyse, wie mit ihnen von Fall zu Fall Beschreibungen erzeugt werden. Zweifelsohne sind viele Beschreibungskontexte und -aufgaben rekurrent, und deshalb finden wir zahlreiche Beschreibungsroutinen, die in der Tat häufig Wortcharakteristik anzunehmen scheinen (in der Weise, dass wiederholt realisierten Handformen in einer Gemeinschaft feste Bedeutungen zugeordnet sind); sie sind Sedimente massiver kultureller Praxis, aus dem Verkehr einer Vielzahl von Individuen entstanden.

Streeck (2013b) stellt die praxeologische Analyse von Gesten so dar:

To investigate gesture in praxeological fashion means to conceive of it, in the first place, as skilled physical praxis, as embodied activity performed according to methods that are shared within some community. Gestures are physical actions by which we „do things“ (Austin 1962) - although the things that gestures do not only include illocutions, but a large, presumably unknown, number of other types of social effects, including directing or attracting attention and showing how something ought to be done. By practices we mean established, common things that get done by gestures, as well as the habitual, routinized methods by which gestures are made. (ebd., S. 674 f.)

The praxeology of gesture takes its cue from Marcel Mauss' notion of techniques of the body, by which the French social anthropologist meant "the ways in which from society to society men know how to use their bodies" (Mauss 1973: 70). [...] Techniques of the body are culturally specific, traditional solutions to recurrent practical, communicative and interaction tasks. But their development in the individual, and as a consequence their manner of use and coordination with other practices, are always also intensely personal affairs. ${ }^{9}$ (ebd., S. $677 \mathrm{f}$.)

\section{Die Wiederkehr des Körpers}

Der Transfer einer sprachlichen Einheit von einer Praktik in eine andere sowie die Ermöglichung und grammatische Sedimentierung einer neuen kommunikativen Praktik durch die Re-Analyse einer sprachlichen Form lassen sich sehr schön an der Re-Analyse der englischen Präposition ,like“ als Fokusmarker und Teil eines verbum dicendi (,be like‘) nachvollziehen. ,Like‘, seit dem späten 18. Jahrhundert auch als Konjunktion im Gebrauch, wird, vermutlich seit den 1950er Jahren in zunehmendem Maße und im Alltagssprachgebrauch selbst von Vierzigjährigen

9 Vgl. Streeck (2013a). 
nunmehr überwiegend auch als Fokusmarker (,And then she made me, like, the sweetest compliment') und seit unbestimmter Zeit auch als Quotativindex (,And I was like, Oh My God!') verwendet. Man kann sich diese Re-Analyse-Geschichte wohl folgendermaßen vorstellen (für eine detailliertere Darstellung siehe Streeck 2002). Die Präposition ,like“ markiert einen Vergleich: Ein Objekt wird im Vergleich zu einem anderen beschrieben. Zu seinem Gebrauch als Fokusmarker mag es gekommen sein, wenn Sprecher den Vergleich anstelle einer direkten Beschreibung benutzen, wenn sie ein Objekt nicht direkt, sondern nur im Vergleich zu einem anderen beschreiben und die Beschreibung dadurch implizit oder gewollt als Beschreibung zweiter Wahl kennzeichnen: ,X ist nicht wirklich P, aber wie P“, ,sozusagen P‘. Mit einer derartigen Markierung wird das Zielelement jedoch unweigerlich auch hervorgehoben, also fokussiert. In dieser Weise wird ,like' oft gebraucht, wie auch im Deutschen ,so‘ und im Ilokano ,kwa' auf diese Weise gebraucht werden. Als Fokusmarker hat ,like“ dieses Kennzeichen von Undeutlichkeit jedoch längst verloren.

Wenn die Beschreibungsobjekte Menschen und ihr Verhalten sind und eine sprachliche Beschreibung unmöglich oder schwierig erscheint, oder auch nur zum Zwecke der Unterhaltung, kann diese auch durch eine mimetische oder pantomimische Inszenierung ersetzt werden: ,He was, like, ( - - ) [folgt szenische Darstellung]‘. Aus diesem Kontext ist das neue verbum dicendi ,be like“ hervorgegangen. Was ,He’s like - she’s like‘ von ,he said - she said“ (vgl. Goodwin 1990) unterscheidet, ist, dass letzteres nur ein sprachliches Zitat zulässt, ersteres jedoch auch ,Körperzitate“ (Streeck 1988a), d.h. Inszenierungen oder Re-Inszenierungen körperlichen Verhaltens: Man kann das Verhalten anderer minutiös und komisch in Szene setzen oder die eigene späte Erkenntnis mit einem Stirnschlag der flachen Hand ,zitieren'. Auf der vorläufig letzten Re-Grammatikalisierungsstufe ist ,be like“ dann zu einem generischen verbum dicendi geworden, das heute sehr viel häufiger als ,say‘ auch einfache Redezitate in Äußerungen verankert. Selbstverständlich ist die grammatische Möglichkeit von ,Körper-Zitaten“ keine Neuheit; in vielen afrikanischen Sprachen etwa sind Ausdrücke, die körperliches Handeln oder körperlichen Ausdruck bezeichnen, die Quelle der Standard-Quotativa (Güldemann 2008), die cline (Hopper/Traugott 1993) führt also von der körperlichen Darstellung zur Sprache. Doch zweifelsohne ist die Verschiebung von ,say zu ,be like‘ ein relativ neues Phänomen, ${ }^{10}$ und damit auch die Re-Konfigurierung

10 Als ich 1989 in den USA zu lehren begann, war dieser Wortgebrauch noch stigmatisiert und galt als typisch für sorority girls, so wie er zuvor schon in Kalifornien als Merkmal von valley girl talk stigmatisiert war. Inzwischen ist es auch für vierzigjährige Männer vollkommen normal ,I'm like - she’s like‘ zu sagen. (Der Name ,Valley Girl Talk‘ wurde durch die Schallplatte Valley Girls 
von Praktiken des Zitierens von rein sprachlichen zu Körperzitaten: Das ,Körperquotativum , be like' subsumiert das sprachliche Zitat gleichsam als einen speziellen Fall, nachdem es vorher durch ,say ' beim Zitieren in eine Nebenrolle gedrängt war.

,Be like‘ subsumiert also die Möglichkeiten von ,say‘, während das umgekehrte nicht der Fall ist: ,He was like‘ kann durch eine körperliche Reinszenierung, durch ein rein sprachliches Zitat oder durch beides gemeinsam vervollständigt werden, während ,say“ allein zitierte Rede zulässt. (Ich sehe hier davon ab, dass solche ,Zitate‘, körperliche wie sprachliche, oft vorgefertigte Formen sind, die eher eine Person charakterisieren, als dass sie Sprechen oder Verhalten ,wörtlich` wiedergeben, wie Tannen 1989 für direkte Rede ausgeführt hat.) Was hier also stattgefunden hat, ist, dass mit einer neuen lexikalischen Einheit eine neue Konstruktion in der Sprache entstanden ist, die Praktiken der Darstellung sozialer, kommunikativer Situationen neu konfiguriert. Mit ihnen werden gleichsam veränderte Präferenzen (im landläufigen, nicht im konversationsanalytischen Sinn) im Sprachsystem verankert, dergestalt, dass bei ,normalem Sprachgebrauch` bei der Wiedergabe erfahrener oder beobachteter kommunikativer Situationen (also bei der ,Redewiedergabe‘) jederzeit körperliche Mimesis, eine ,schauspielernde“ Darbietung des Geschehens, möglich ist.

Dass diese Entwicklung in mehreren Sprachgemeinschaften stattgefunden zu haben oder stattzufinden scheint, ${ }^{11}$ wäre einer Untersuchung wert; sollte sich die Vermutung bestätigen, spräche dies dafür, dass entweder in den Industriegesellschaften neue kommunikative Aufgaben entstanden sind, vermutlich zunächst für jüngere Menschen, oder dass sich durch internationale Kontakte neue (oder erneuerte) kommunikative Praktiken verbreiten. Natürlich sind ,Körperzitate“ nichts Neues, aber dass ihnen gleichsam von der offiziellen Seite der Grammatik so viel Raum gegeben wird, scheint in meiner eigenen Lebenszeit neu zu sein. (Vergleiche damit die offenkundig viel ältere ,Grammatikalisierung‘ beschreibenden Gestikulierens durch ,so; siehe oben.) Interessant ist daran nicht zuletzt die Privilegierung strikt ,mündlicher‘ Formate: Redezitate können jederzeit in Schrift übersetzt werden, Körperzitate nur sehr selten.

Welches die kommunikativen Aufgaben sind, auf die ,be like‘ und seine Analoga in anderen Sprachen zunächst geantwortet haben, lässt sich nur vemuten. Ein Redekontext, der ganz und gar nicht ohne Mimesis auskommt, ist der, dass

von Moon Unit Zappa bekannt. Das ,Valley` ist das San Fernando Valley in den Suburbs von Los Angeles.)

11 Für das Deutsche hat dies z.B. Golato (2000) dokumentiert; für das Französische und Italienische stütze ich mich auf mündliche Berichte. 
sich Kinder von Filmen (Actionfilmen) erzählen: Hier kommt dem Dialog der Handlungen und den sound effects besondere Bedeutung zu, und die kann man nicht mit he said she said zitieren. Hierfür ist das folgende ein typisches Beispiel: Zwei zehnjährige Jungen sprechen über die Filme „Alien“ und „Aliens“ (vgl. Streeck 2002 für eine genauere Analyse). Hier wird zwar ,be like‘ nicht als verbum dicendi benutzt, aber es wird deutlich, wie es zitierte Handlungen markiert (Z. 01 und 05).

(8)

$01 \mathrm{~J}$ Well the alien, like, sticks this ( - - ) long spike into his back?

Also der Alien, [like], stösst diesen ( - - ) langen Zacken in seinen Rücken?

02

It's like, rips him open

Also so, reisst ihn auf

Wurfgeste

03

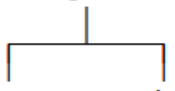

throws him up

wirft ihn hoch

04

and rips him apart?

und reisst ihn in Stücke?

05

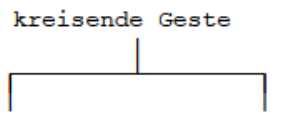

And he's like- sliding on the floor.

Und er [like-] rutsch so auf dem Boden rum.

In der Geschichte von ,like‘ hören wir das ironische Gelächter der Sprachgeschichte und erleben die Wiederkehr des Körpers (vgl. Kamper/Wulf (Hg.) 1982), wenn nicht gar die Auferstehung der Toten. Denn ,like‘ stammt ja von einem (proto-germanischen) Wort ab, das nichts anderes bedeutet als Körper - ,IIk (,liek ${ }^{`}$ gesprochen) - und im Deutschen als ,Leiche“ überlebt hat. ${ }^{12}$

12 Doch wie kam man von ,Körper‘ zum temporalen, gleich‘? 


\section{Zusammenfassung}

In diesem Beitrag habe ich dargestellt, wie sich der Charakter meiner Forschung zur Gestik, beginnend mit einem unerwarteten finding zum Blickverhalten von Sprechern beim Gestikulieren, allmählich von dem Versuch, gestische Formen zu identifizieren und ihre Funktionen in sprachlichen Äußerungen zu bestimmen, in eine Forschung über gestische und sprachliche Praktiken verwandelt hat. Ich habe versucht zu zeigen, dass verschiedene sprachliche Formen und Konstruktionen, die im Zusammenhang mit Gesten realisiert werden, als Sedimente kommunikativer Praktiken begriffen werden müssen. Hierbei handelte es sich in erster Linie um die Form ,so‘ und ihr japanisches Äquivalent ,ko', um das Ilokano,Verb' ,(i-)kastoy“, , so (von sich weg) machen', und das neue US-Englische verbum dicendi ,be like'. Bei ,be like', dessen Entstehung noch nicht sehr lange zurückliegt, kann man diesen Prozess unschwer rekonstruieren. Seine Sedimentierung ist Teil einer offenbar in mehreren Sprachgemeinschaften stattfindenden Rekonfigurierung von Praktiken des Zitierens, in der sprachliche Zitate gewissermaßen als Spezialfall von körperlichen Re-Inszenierungen gefasst werden und nicht umgekehrt wie zuvor die körperliche, pantomimische Reinszenierung des Verhaltens des zitierten Menschen als Beiwerk zur Redewiedergabe behandelt wird.

Gestische Beschreibungen finden sich oft im Kontext von Wortsuchen, wo sie mit anderen praxisspezifischen Einheiten oder Formen interagieren, z.B. dem Ilokano-Wortsuchenwort ,kwa und der suche-markierenden Wiederholung von Funktionswörtern oder Morphemen. So, wie sich die Praktiken des Suchens, gestischen Beschreibens und der Fokussierung überlappen, so überlappen sich auch diese Formen: ,so', ,kwa', like‘ usw. können auch als Fokusmarker dienen. Nur wenn das gleichzeitige körperliche Handeln des Sprechers in Rechnung gestellt wird, lassen sich die Funktionen unterscheiden. Will man die kommunikativen Prozesse des menschlichen Gesprächs also auf kohärente Weise in ihre signifikanten Bestandteile zerlegen und deren Funktionen bestimmen, so muss man wiederkehrende Formen (Einheiten und die Konstruktionen, in denen sie vorkommen) als Produkte und Ressourcen kommunikativer Praxis fassen, die körperlichen Ausdruck einschließt. Die Habitualisierung von Praxis in der Form von Praktiken - also habitualisierten Methoden, mit denen einzelne Handlungen realisiert werden - hat logische Priorität gegenüber der Konventionalisierung von Formen, und manche Modi der Kommunikation, z.B. das gestische Beschreiben, lassen gar keine andere Beschreibung als eine praxeologische $\mathrm{zu}^{13}$

13 Dies heißt nicht, dass jede kommunikative Handlung eine Praktik realisiert; sie kann auch improvisieren, eine Praktik modifizieren etc. 


\section{Literatur}

Arnheim, Rudolf (1969): Visual thinking. Berkeley.

Austin, John L. (1962): How to do things with words. Oxford.

Donald, Merlin (1991): Origins of the modern mind: Three stages in the evolution of culture and cognition. Cambridge, MA.

Goffman, Erving (1981): Forms of talk. Oxford.

Golato, Andrea (2000): Und ich so/und er so [and l'm like/and he's like]: An innovative German quotative for reporting on embodied actions. In: Journal of Pragmatics 32, S. 29-54.

Goodman, Nelson (1976): Languages of art. An approach to a theory of symbols. 2. Aufl. Indianapolis. [Erstveröffentlichung Indianapolis 1968.]

Goodwin, Marjorie Harness (1990): He-Said-She-Said. Talk as social organization among black children. Bloomington.

Gulldemann, Tom (2008): Quotative indexes in African languages. A synchronic and diachronic survey. (= Empirical Approaches to Language Typology 34). Berlin.

Gullberg, Marianne/Holmqvist, Kenneth (1999): Keeping an eye on gestures: Visual perception of gestures in face-to-face communication. In: Pragmatics and Cognition 7 (1), S. 35-63.

Hole, Daniel/Klumpp, Gersson (2000): Definite type and indefinite token: The article son in colloquial German. In: Linguistische Berichte 182, S. 231-244.

Hopper, Paul J./Traugott, Elizabeth C. (1993): Grammaticalization. Cambridge.

Kallmeyer, Werner/Schmitt, Reinhold (1996): Forcieren oder: Die verschärfte Gangart. Zur Analyse von Kooperationsformen im Gesprăch. In: Kallmeyer, Werner (Hg.): Gesprăchsrhetorik: Rhetorische Verfahren im Gesprächsprozess. (= Studien zur Deutschen Sprache 4). Tübingen, S. 19-118.

Kamper, Dietmar/Wulf, Christoph (Hg.) (1982): Die Wiederkehr des Körpers. Frankfurt a.M.

Kendon, Adam (1980): Gesticulation and speech: Two aspects of the process of utterance. In: Key, Mary Ritchie (Hg.): The relationship of verbal and nonverbal communication. (= Contributions to the Sociology of Language 25). Den Haag, S. 207-227.

Kendon, Adam (2004): Gesture: Visible action as utterance. Cambridge.

Knöbl, Ralf (2014): Variation im Standard. Formale und funktionale Variationsaspekte des gesprochensprachlichen Gebrauchs indefiniter Referenzierungsformen. In: Bühler, Rudolf/ Bürkle, Rebekka/Leonhardt, Nina Kim (Hg.): Sprachkultur - Regionalkultur. Neue Felder kulturwissenschaftlicher Dialektforschung. (= Studien und Materialien des LudwigUhland-Instituts der Universităt Tubingen 49). Tubingen, S. 154-185.

Mauss, Marcel (1973): The techniques of the body. In: Economy and Society 2, S. 70-88. [Erstveröffentlichung 1935 in Journal de Psychologie Normal et Pathologique 32, S. 271-293].

Sauer, Beverly J. (2003): The rhetoric of risk. Technical documentation in hazardous environments. Mahwah, NJ.

Schegloff, Emanuel A. (1984): On some gestures' relation to talk. In: Atkinson, John Maxwell/ Heritage, John ( $\mathrm{Hg}$.): Structures of social action. (= Studies in Emotion and Social Interaction). Cambridge, S. 266-295.

Sowa, Timo (2006): Understanding coverbal iconic gestures in shape descriptions. (= Dissertationen zur künstlichen Intelligenz 294). Berlin.

Streeck, Jürgen (1988a): Körperzitate („Body Quotes“). Vortrag in der AG „Interpretative Soziolinguistik von Sprechstilen“ bei der 10. Jahrestagung der Deutschen Gesellschaft für Sprachwissenschaft, Gesamthochschule Wuppertal, 2.-4. Mărz 1988. 
Streeck, Jürgen (1988b): The significance of gesture: How it is established. In: Papers in Pragmatics 2, S. 60-83.

Streeck, Jürgen (1993): Gesture as communication I: Its coordination with gaze and speech. In: Communication Monographs 60, S. 275-299.

Streeck, Jürgen (1995): On projection. In: Goody, Esther N. (Hg.): Social intelligence and interaction: Expressions and implications of the social bias in human intelligence. Cambridge, S. 87-110.

Streeck, Jürgen (1996): A little Ilokano grammar as it appears in interaction. In: Journal of Pragmatics 26, S. 189-213.

Streeck, Jürgen (2002): Grammars, words, and embodied meanings. On the evolution and uses of so and like. In: Journal of Communication 52, S. 581-596.

Streeck, Jürgen (2008): Depicting by gestures. In: Gesture 8, S. 285-301.

Streeck, Jürgen (2009): Gesturecraft. The manu-facture of meaning. (= Gesture Studies 2). Amsterdam.

Streeck, Jürgen (2013a): Interaction and the living body. In: Journal of Pragmatics 46, S. 69-90.

Streeck, Jürgen (2013b): Praxeology of gesture. In: Muller, Cornelia et al. (Hg.): Body, language, communication. An international handbook on multimodality in human interaction.

1. Halbbd. (= Handbücher zur Sprach- und Kommunikationswissenschaft 38.1). Berlin, S. $674-685$.

Stukenbrock, Anja (2010): Oberlegungen zu einem multimodalen Verständnis gesprochener Sprache am Beispiel deiktischer Verwendungsweisen des Ausdrucks ,so'. In: Dittmar, Norbert/Bahlo, Nils (Hg.): Beschreibungen für gesprochenes Deutsch auf dem Prüfstand. Analysen und Perspektiven. (= Deutsche Sprachwissenschaft international 11). Frankfurt a.M., S. 165-193.

Stukenbrock, Anja (2015): Deixis in der Face-to-Face Interaktion. Berlin.

Tannen, Deborah (1989): Talking voices. Repetition, dialogue, and imagery in conversational discourse. (= Studies in Interactional Sociolinguistics 6). Cambridge.

von Heusinger, Klaus (2012): Referentialităt, Spezifizităt, und Diskursprominenz im Sprachvergleich. In: Gunkel, Lutz/Zifonun, Gisela (Hg.): Deutsch im Sprachvergleich: Grammatische Kontraste und Konvergenzen. (= Jahrbuch des Instituts für Deutsche Sprache 2011). Berlin, S. 417-455.

Wiese, Heike (2011): So as a focus marker in German. In: Linguistics 49, S. 991-1039.

Zima, Elisabeth (2014): Gibt es multimodale Konstruktionen? Eine Studie zu [V(motion) in circles] und [all the way from X PREP Y]. In: Gesprächsforschung 15, S. 1-48. Internet: www.gespraechsforschung-0zs.de/fileadmin/dateien/heft2014/ga-zima.pdf [Stand: 10.8.2015]. 
